\title{
Prediction of Wine Quality Using Machine Learning Algorithms
}

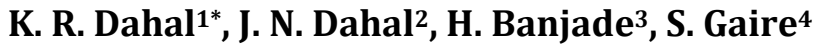 \\ ${ }^{1}$ Department of Statistics, Truman State University, Kirksville, MO, USA \\ ${ }^{2}$ Department of Physics, Virginia Union University, Richmond, VA, USA \\ ${ }^{3}$ Department of Physics, Virginia Commonwealth University, Richmond, VA, USA \\ ${ }^{4}$ Department of Physics, The Catholic University of America, Washington D. C., USA \\ Email: ^kdahal@truman.edu
}

How to cite this paper: Dahal, K.R., Dahal, J.N., Banjade, H. and Gaire, S. (2021) Prediction of Wine Quality Using Machine Learning Algorithms. Open Journal of Statistics, 11, 278-289.

https://doi.org/10.4236/ojs.2021.112015

Received: February 9, 2021

Accepted: March 15, 2021

Published: March 18, 2021

Copyright (c) 2021 by author(s) and Scientific Research Publishing Inc. This work is licensed under the Creative Commons Attribution International License (CC BY 4.0).

http://creativecommons.org/licenses/by/4.0/

(cc) (i) Open Access

\begin{abstract}
As a subfield of Artificial Intelligence (AI), Machine Learning (ML) aims to understand the structure of the data and fit it into models, which later can be used in unseen data to achieve the desired task. ML has been widely used in various sectors such as in Businesses, Medicine, Astrophysics, and many other scientific problems. Inspired by the success of ML in different sectors, here, we use it to predict the wine quality based on the various parameters. Among various ML models, we compare the performance of Ridge Regression (RR), Support Vector Machine (SVM), Gradient Boosting Regressor (GBR), and multi-layer Artificial Neural Network (ANN) to predict the wine quality. Multiple parameters that determine the wine quality are analyzed. Our analysis shows that GBR surpasses all other models' performance with MSE, R, and MAPE of $0.3741,0.6057$, and 0.0873 respectively. This work demonstrates, how statistical analysis can be used to identify the components that mainly control the wine quality prior to the production. This will help wine manufacturer to control the quality prior to the wine production.
\end{abstract}

\section{Keywords}

Wine Quality, Neural Network, Machine Learning (ML), Artificial Intelligence (AI)

\section{Introduction}

Wine is the most commonly used beverage globally, and its values are considered important in society. Quality of the wine is always important for its consumers, and mainly for producers in the present competitive market to raise the 
revenue. Historically, wine quality used to be determined by testing at the end of the production; to reach that level, one already spends lots of time and money. If the quality is not good, then the various procedure needs to be implemented from the beginning, which is very costly. Every person has their own opinion about the taste, so identifying a quality based on a person's taste is challenging. With the development of technology, the manufacturers started to rely on various devices for testing in development phases. So, they can have a better idea about wine quality, which, of course, saves lots of money and time. In addition, this helped in accumulating lots of data with various parameters such as quantity of different chemicals and temperature used during the production, and the quality of the wine produced. These data are available in various databases (UCL Machine Learning Repository, and Kaggle). With the rise of ML techniques and their success in the past decade, there have been various efforts in determining wine quality by using the available data [1] [2] [3]. During this process, one can tune the parameters that directly control the wine quality. This gives the manufacturer a better idea to tune the wine quality by tuning different parameters in the development process. Besides, this may result in wines with multiple tastes, and at last, may result in a new brand. Hence, the analysis of the basic parameters that determine the wine quality is essential. In addition to humanitarian efforts, ML can be an alternative to identify the most important parameters that control the wine quality. In this work, we have shown how ML can be used to identify the best parameter on which the wine quality depends and in turn predict wine quality.

Our work is organized as follows: In Section 2, we discuss data description and preprocessing of the dataset used in this work. In Section 3, we briefly discuss the proposed methodology, followed by model comparison and selection of best model in Section 4. In Section 5, we summarize the main finding and conclusion.

\section{Data Description and Preprocessing}

\subsection{Data Source and Description}

In this study, we use the publicly available wine quality dataset obtained from the UCL Machine Learning Repository, which contains a large collection of datasets that have been widely used by the machine learning community [4]. Among the two types of wine quality dataset (redwine and white wine), we have chosen redwine data for our study because of its popularity over the white wine. The redwine dataset contains 11 physiochemical properties: fixed acidity $\left(\mathrm{g}[\right.$ tartaric acid $\left.] / \mathrm{dm}^{3}\right)$,volatile acidity $\left(\mathrm{g}[\right.$ acetic acid $\left.] / \mathrm{dm}^{3}\right)$, total sulfur dioxide $\left(\mathrm{mg} / \mathrm{dm}^{3}\right)$, chlorides $\left(\mathrm{g}\left[\right.\right.$ sodium chloride] $\left./ \mathrm{dm}^{3}\right), \mathrm{pH}$ level, free sulfur dioxide $\left(\mathrm{mg} / \mathrm{dm}^{3}\right)$, density $\left(\mathrm{g} / \mathrm{cm}^{3}\right)$, residual sugar $\left(\mathrm{g} / \mathrm{dm}^{3}\right)$, citric acid $\left(\mathrm{g} / \mathrm{dm}^{3}\right)$, sulphates (g[potassium sulphate $] / \mathrm{dm}^{3}$ ), and alcohol (vol\%). Alongside these properties, a sensory score was acquired from several different blind taste testers which graded each wine sample with a score ranging from zero (poor) to 10 (excellent). The 
median was recorded and serves as the response variable [5]. The dataset contains the records of 4898 random samples of wine manufactured. Various statistical analyses were done to understand the nature of the dataset as presented in Table 1.

The Pearson correlation coefficient ( $r$ ) measures the strength of the association between two different variables. The association between two variables is considered highly positive if ' $r$ ' is close to 1 while highly negative if " $r$ " is close to -1 . Before passing the data into the ML models, we calculated the Pearson correlation coefficient between each variable and the wine quality (i.e., target property) in our dataset, as presented in Table 2. Our analysis shows that quantity of alcohol has the highest $(0.435)$, while the citric acid has the lowest $(-0.009)$ correlation coefficients with the target property. The variables which have the significantly lower correlation coefficient (close to zero) with the target property can be considered as irrelevant in the statistical analysis. While training the ML models these variables can have significant effect in the predicted property, as they introduced the noise in the dataset and mislead the training process. This results in poor models and less accurate prediction performance. There are different ways to decrease noise [6]. One of the most popular and commonly used methods of denoising is dropping the irrelevant, redundant, and insignificant predictors. The method, which is simple, and convenient comes first in the mind of a statistician [7] [8].

ML algorithms are sensitive to the outliers. It can spoil and mislead the training process. As a result, we may end up with poor models which ultimately give less accurate results. So, it is customary to check outliers during the data preprocessing. A boxplot is a standardized way of displaying the distribution of the

Table 1. Descriptive statistics of the variables of the redwine data.

\begin{tabular}{cccccc}
\hline Variable Name & Mean & $\begin{array}{r}\text { Standard } \\
\text { deviation }\end{array}$ & Minimum & Maximum & Median \\
\hline Fixed acidity & 6.854 & 0.843 & 3.80 & 14.2 & 6.80 \\
Volatile acidity & 0.278 & 0.100 & 0.08 & 1.10 & 0.26 \\
Citric acid & 0.334 & 0.121 & 0.00 & 1.66 & 0.32 \\
Residual sugar & 6.391 & 5.072 & 0.60 & 65.8 & 5.20 \\
Chlorides & 0.045 & 0.021 & 0.009 & 0.35 & 0.04 \\
Free sulfur dioxide & 35.30 & 17.00 & 2.00 & 289 & 34.0 \\
Total sulfur dioxide & 138.4 & 42.49 & 9.00 & 440 & 134 \\
Density & 0.994 & 0.002 & 0.99 & 1.038 & 0.99 \\
PH & 3.188 & 0.151 & 2.27 & 3.82 & 3.18 \\
Sulphates & 0.489 & 0.114 & 0.22 & 1.08 & 0.47 \\
Alcohol & 10.51 & 1.230 & 8.00 & 14.2 & 6.00 \\
\hline Quality & 5.877 & 0.885 & 3.00 & & \\
\hline
\end{tabular}


Table 2. The value of the pearson correlation coefficient $(\boldsymbol{r})$ of the predictors with respect to the target variable: quality.

\begin{tabular}{cccccr}
\hline Predictor & $\boldsymbol{r}$ & Predictor & $\boldsymbol{r}$ & Predictor & \multicolumn{1}{c}{$\boldsymbol{r}$} \\
\hline Alcohol & 0.435 & Citric acid & -0.009 & Volatile acidity & -0.194 \\
$\mathrm{pH}$ & 0.099 & Residual sugar & -0.097 & Chlorides & -0.209 \\
Sulphates & 0.053 & Fixed acidity & -0.113 & Density & -0.307 \\
Free sulfur dioxide & 0.008 & Total sulfur dioxide & -0.174 & & \\
\hline
\end{tabular}

data. It is commonly used to identify the shape of the distribution and the possible existence of the outliers. Boxplots of each feature are plotted in Figure 1. Based on these boxplots, all the variables except alcohol are either skewed or possibly contain outliers. To get rid of outliers, we may drop the extreme values from the data set. However, dropping data is always a harsh step, so should be taken only in the extreme condition when we are $100 \%$ sure that the outliers are the measurement errors. At this point, we are unable to drop these extreme values because we are unable to confirm these extreme values as measurement errors.

\subsection{Feature Scaling}

As presented in Table 1, the variables are spread widely. For instance, the values of total Sulphur dioxide are extremely large compared to the chlorides. Such a large value of one variable can have dominance over other quantities during the training process in ML models. For instance, while doing K-nearest neighbor KNN [9], or SVM if one does not standardize the nonuniform data, the datapoints with high distance will dominate the performance of the KNN or SVM model. So, feature scaling is a very important step one need to take care of, before training any ML model. There are many feature scaling methods. The most common and popular techniques that have been using in the ML community are standardization and normalization. There is not theoretical evidence of claiming which method work best. To scale the features of the dataset, standardization has been used. The formulas used to calculate the standardization is as follows:

$$
z=\frac{x-\text { mean }}{\text { std }}
$$

where $z, x$, mean, and $s t d$ are standardized input, input, mean and standard deviation of the feature, respectively.

\subsection{Data Partition}

The data was split into training data set and testing data set in the ratio $3: 1$. We train data and is used to find the relationship between target and predictor variables. The main purpose of the splitting data is to avoid overfitting. If overfitting occurs, the machine learning algorithm could perform exceptionally in the training dataset, but perform poorly in the testing dataset [10]. 


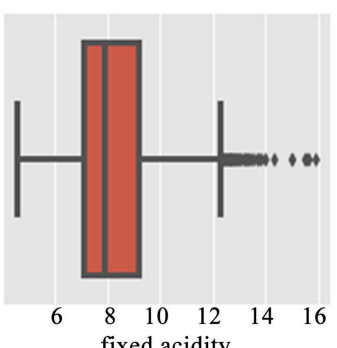
fixed acidity

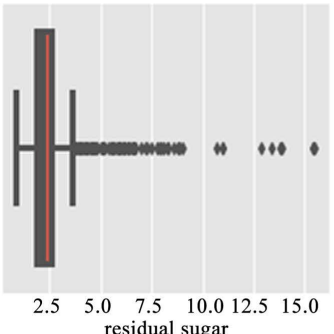
sugar

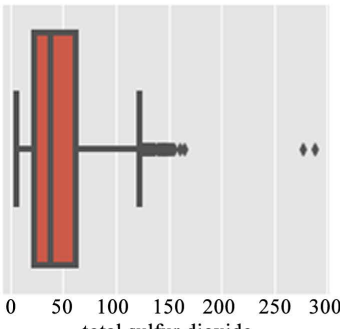
total sulfur dioxide

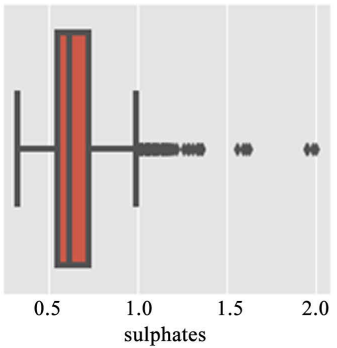

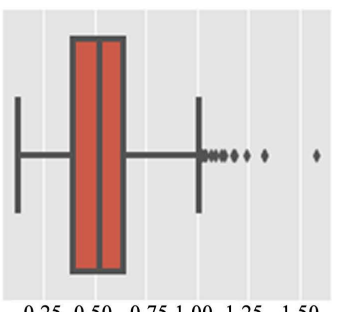

$\begin{array}{llllll}0.25 & 0.50 & 0.75 & 1.00 & 1.25 & 1.50\end{array}$ volatile acidity

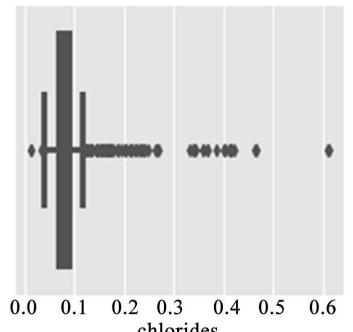

chlorides

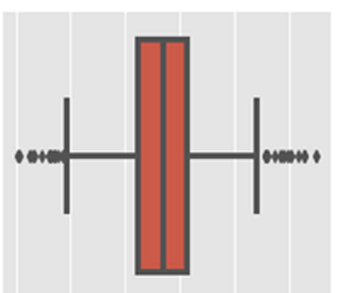

0.99000 .99250 .99500 .99751 .00001 .0025 density

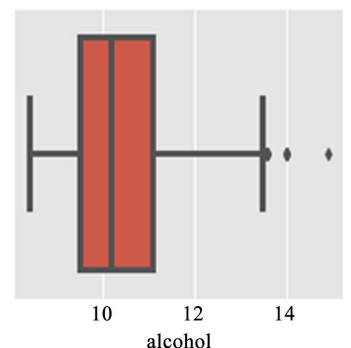

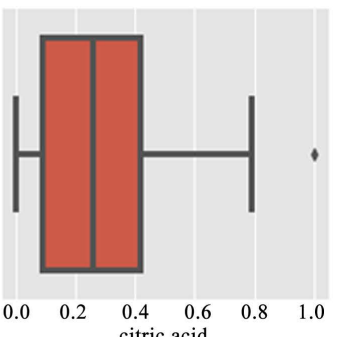

citric acid
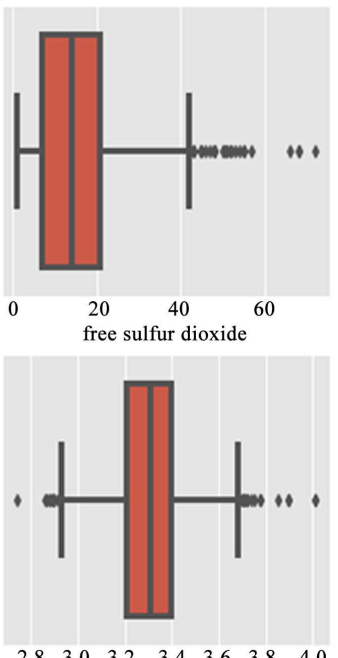

$\mathrm{pH}$

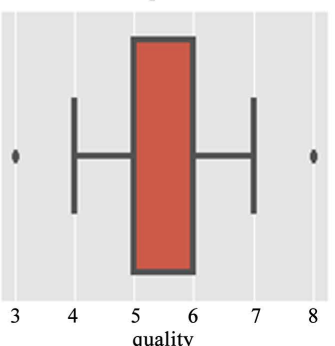

Figure 1. Box plot of the variables of the redwine data.

\section{Machine Learning Algorithms}

A wide range of machine learning algorithms such as linear regression, logistic regression, support vector machine, and kernel methods, neural networks, and many others are available for the learning process [11]. Each technique has its strength and weakness. In this work, we use the following supervised learning algorithms to predict wine quality.

\subsection{Ridge Regression}

Ridge Regression (RR) is very similar to the multiple linear regression. In the multiple linear regression, the parameters $\beta_{j}$ are estimated by minimizing residual sum of squares (RSS) defined in Equation (2).

$$
\mathrm{RSS}=\sum_{i=1}^{n}\left(\left(y_{i}-\beta_{0}-\sum_{j=1}^{p} \beta_{j} x_{i j}\right)\right)^{2}
$$

where $y_{i}$ are the observed value, and $x_{i j}$ are the predictors. 
In the $\mathrm{RR}$, the parameters are $\beta_{j}$, the values that minimizes

$$
\sum_{i=1}^{n}\left(\left(y_{i}-\beta_{0}-\sum_{j=1}^{p} \beta_{j} x_{i j}\right)\right)^{2}+\lambda \sum_{j=1}^{p} \beta_{j}^{2}=\mathrm{RSS}+\lambda \sum_{j=1}^{p} \beta_{j}^{2}
$$

where $\lambda \sum_{j=1}^{p} \beta_{j}^{2}$ is called the shrinkage penalty and $\lambda$ is the tuning parameter of the model [12]. When $\lambda=0$, the RR is the same as linear regression because of having common parameters. For the small value of $\lambda$, there is not a significant difference between the parameters of the models. As $\lambda$ increases, the parameters of the RR started to shrink and converge to zero as $\lambda \rightarrow \infty$. The value of $\lambda$ plays a crucial role in the model performance. When $\lambda$ is small there is high variance and low bias; the model outperforms in the training set, while it has poor performance in the unseen data, which results in overfitting. When $\lambda$ increase, the variance decreased, and the bias increases. For the sufficient high value of $\lambda$, there might be underfitting, so a good choice of $\lambda$ can have a best model, with best prediction performance.

\subsection{Support Vector Machine}

Support Vector Machine (SVM) is one of the most popular and powerful machine learning algorithms which was introduced in the early 90s. When used for regression, SVM is also known as Support Vector Regressor (SVR). SVR is a kernel-based regression technique which maps nonlinearly separable data in real space to higher dimension space using kernel function [13]. It is equipped with various kernels such as linear, sigmoid, radial, and polynomial. In this work, we have used radial basis kernel (RBF) because it outperformed other kernels based SVR in redwine dataset. The performance of the SVR is controlled by two important tuning parameters (cost: regularization parameter and gamma: kernel coefficient for RBF). The tuning parameter cost control the bias and variance trade-off. The small value of the tuning parameters cost underfits the data, whereas the large value overfit [12].

\subsection{Gradient Boosting Regressor}

Gradient Boosting Regression (GBR) is one of the leading ensemble algorithms used for both classification and regression problems. Which builds an ensemble of weak learners in sequence with each tree and together make an accurate predictor. Decision tree is one of the most popular choice of such ensemble models. Each new tree added to the ensemble model (combination of all the previous tree) minimize the loss function associated with the ensemble model. The loss function depends on the type of the task performed and can be chosen by the user. For GBR, the standard choice is the squared loss. A key factor of this model is that adding sequentially trees that minimize the loss function, the overall prediction error decreases [14] [15]. By tuning many hyperparameters such as the learning rate, the number of trees, maximum depth we can control the gradient boosting performance which helps to make model fast and less complex. Detailed explanation of the GBR algorithm can be found in Friedman et al. [14]. 


\subsection{Artificial Neural Network (ANNs)}

ANNs are a very primitive generalization of biological neurons. They are composed of layers of computational units called neurons, with a connection between different layers through the adjustable weights. The major constituents of ANNs are weights, bias, and the activation function. An excellent choice of the activation function results in the proper accuracy of an ANN model. The most widely used activation functions are Logistic (known as Sigmoid) [16] [17] Rectified linear unit, [18] and the SoftPlus [19]. Passage of information along a predetermined path between the neurons is the fundamental idea behind the construction of ANNs. Its architecture is very flexible, and various network parameters (such as weights, bias, number of nodes, and number of hidden layers) can be tuned to improve the performance of the network. One can add up the information from multiple sources to the neurons and apply a non-linear transformation at each node, which helps the network to learn the complexity present in the data. With the application of linear and non-linear transformation in the input data, ANNs transform those initial representations up to a specific outcome. Depending on the learning task, the outcome of the network could be either classification or regression. The schematic diagram for the ANN used in this work is presented in Figure 2.

\section{Results and Discussion}

With the goal of assessing the performance of the different ML algorithms, we have used four most popular machine learning algorithms, namely: Ridge Regression (RR), Support Vector Machine (SVM), Gradient Boosting Regressor (GBR), and Artificial Neural Network (ANN) to predict the wine quality in the redwine data. This allows us the freedom to select the most suitable ML algorithm to predict the wine quality with the given variables.

All of the model's performance (on the training and test data) explained in the previous sections are evaluated by using Mean Squared Error (MSE), Mean Absolute Percentage Error (MAPE), and correlation coefficient $(R)$ defined as

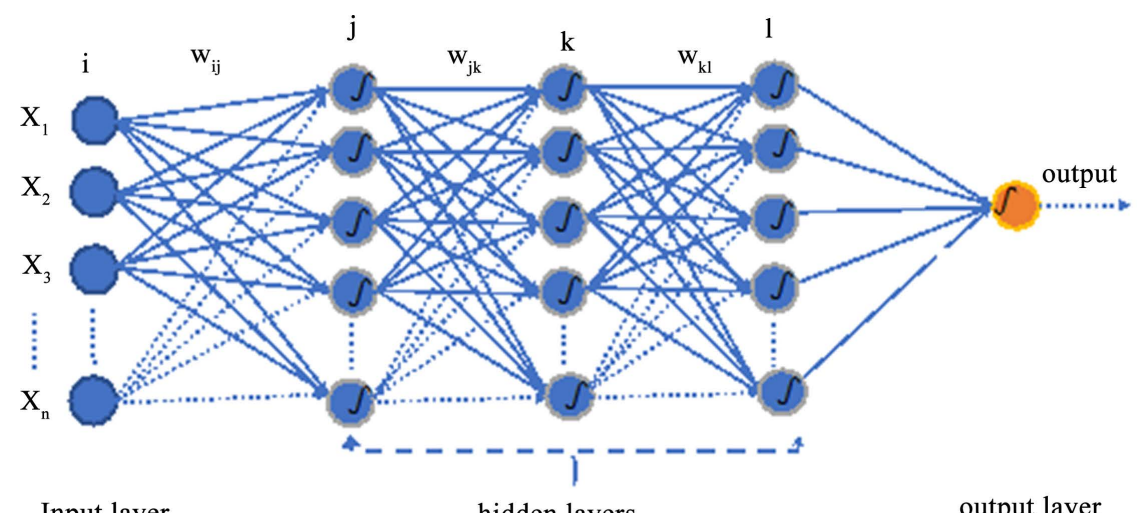

Input layer

...hidden layers ...

output layer

Figure 2. The schematic of an ANN, with three hidden layers and one output layer with Relu activation function at each node where $w_{i j}, w_{j k}$, and $w_{k l}$ are the weights. 


$$
\begin{gathered}
\text { MSE }=\frac{1}{n} \sum_{i=1}^{n}\left(y_{i}-\widehat{y_{i}}\right)^{2} \\
\operatorname{MAPE}=\frac{1}{n} \sum_{i=1}^{n}\left|\frac{y_{i}-\widehat{y_{i}}}{y_{i}}\right| \\
R=\frac{\sum_{i=1}^{n}\left(y_{i}-\widehat{y_{i}}\right)\left(\widehat{y_{i}}-\widehat{\widehat{y_{i}}}\right)}{\sqrt{\sum_{i=1}^{n}\left(y_{i}-\widehat{y_{i}}\right)^{2}\left(\widehat{y_{i}}-\widehat{y_{i}}\right)^{2}}}
\end{gathered}
$$

where, $y_{i}$ and $\widehat{y_{i}}$ are the target observed and target predicted values respectively. And $\mathrm{n}$ is the total number of observations.

The 10 -fold cross-validation is used to choose the best tuning parameter $\lambda$ for the RR model. We used the grid search technique to obtain the optimal parameter by varying $\lambda$ from 0.00001 to 100 with an increment of 0.01 . Figure 3 shows the variation in MSE with $\lambda$. We obtained the optimal value of $\lambda$ as 45.25 , that minimizes the MSE. A RR model using this tuning parameter $\lambda$ is fitted, and its performance is presented in Table 3.

In addition to the $\mathrm{RR}$, the performance of the kernel-based machine learning algorithm SVM is compared. Similar to RR, the 10-fold cross-validation is used to obtain the tuning parameters cost and gamma. We used the grid search technique to obtain these tuning parameters by varying each between 0.01 to 10 . For each possible combination of cost and gamma, MSE is computed. A heatmap of

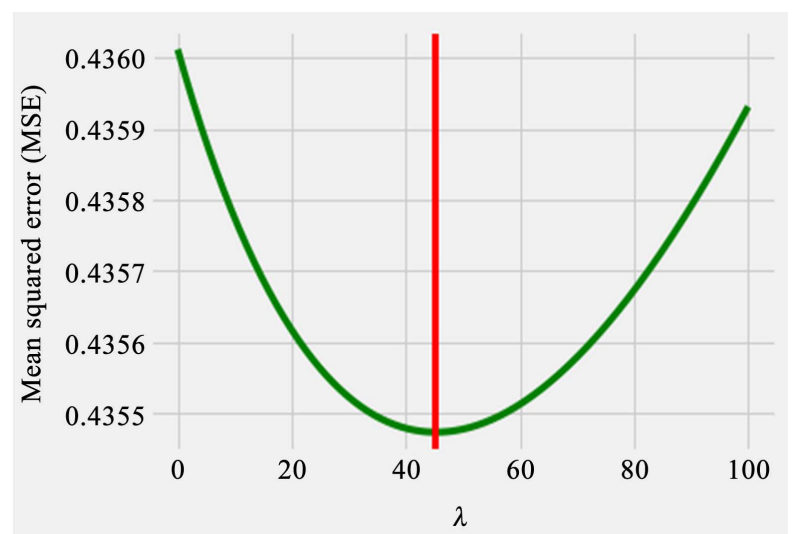

Figure 3. Graph of tuning parameter $\lambda$ versus the Mean squared error (MSE) of the RR model.

Table 3. Model performance metrics obtained using training and test datasets.

\begin{tabular}{ccccccc}
\hline \multirow{2}{*}{ Models } & \multicolumn{3}{c}{ Training data set } & \multicolumn{3}{c}{ Test data set } \\
\cline { 2 - 7 } & $\boldsymbol{R}$ & MSE & MAPE & $\boldsymbol{R}$ & MSE & MAPE \\
\hline RR & 0.6029 & 0.4281 & 0.0934 & 0.5897 & 0.3869 & 0.0888 \\
SVM & 0.7797 & 0.267 & 0.1426 & 0.5971 & 0.3862 & 0.1355 \\
GBR & 0.7255 & 0.3286 & 0.0826 & 0.6057 & 0.3741 & 0.0873 \\
ANN & 0.66 & 0.37 & 0.14 & 0.58 & 0.4 & 0.12 \\
\hline
\end{tabular}


these tuning parameters versus MSE is plotted in Figure 4. The optimal values the parameters computed using 10 -fold cross-validation are cost $=0.95$ and gamma $=0.13$. An SVM model with these tuning parameters is fitted, and its performance is presented in Table 3.

Gradient boosting was also used to predict the wine quality. It has hyperparameters to control the growth of Decision Trees (e.g., max_depth, learning rate), as well as hyperparameters to control the ensemble training, such as the number of trees (n_estimators). In the tuning of the model parameters, we test the learning rate from low (0.01) to high (0.2) as well as a number of trees in the range 1 to 200. The results show that setting the learning rate to (0.05) has better predictive outcomes. Figure 5 shows the change in validation error with the number of iterations. We use an early stopping process that performs model optimization by monitoring the model's performance on a separate test data set and stopping the training procedure once the performance on the test data stops improving beyond a certain number of iterations. We found better predictive outcomes at n_estimators $=40$, which is indicated by a red star in Figure 5 . The GBR model based on this tuning parameter is fitted, and its performance is presented in Table 3.

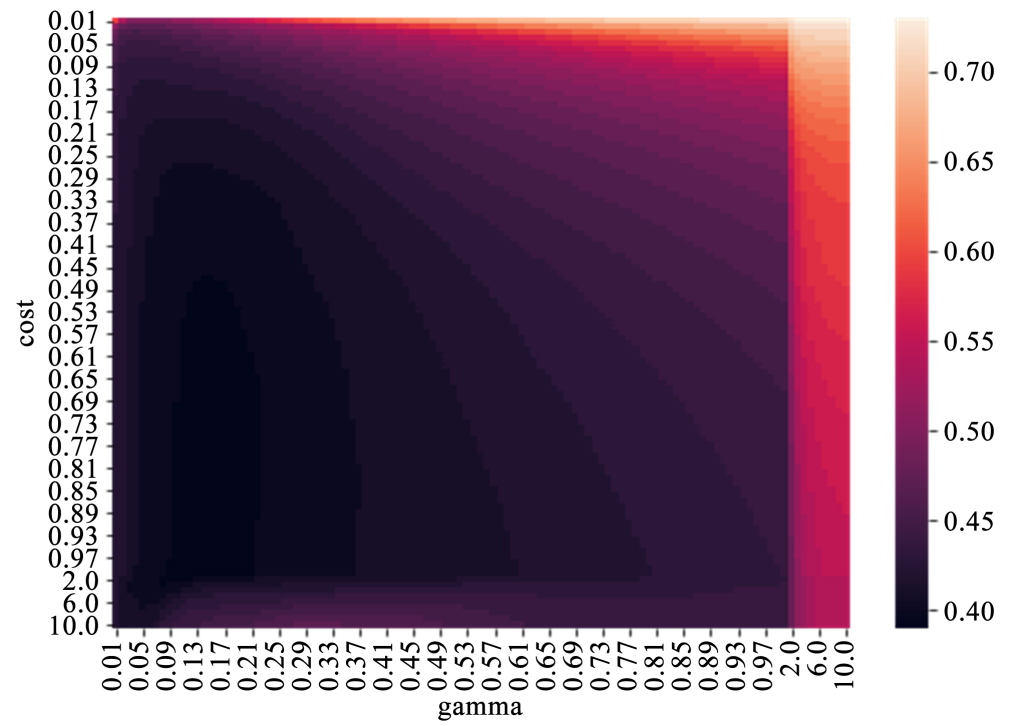

Figure 4. Heatmap showing tuning parameters cost and gamma with colors bars displaying mean squared error.

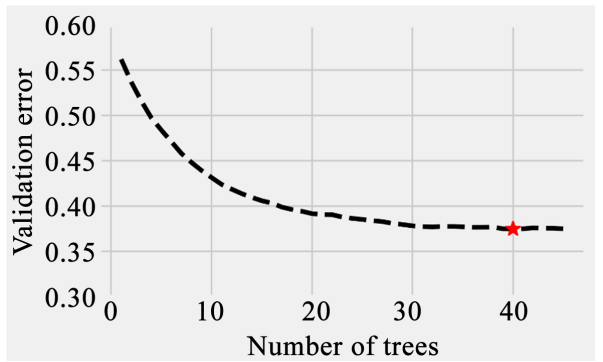

Figure 5. Variation of validation error with number of trees (i.e. n_estimators). 
As ANN performs very well in compared to other mathematical models in most of the dataset, we test its performance to predict the wine quality in redwine dataset. Before using the model in the test dataset, we train the model by tuning various network parameters such as the number of layers and the number of nodes in each layer. For the sake of comparison, we used gradient descent (GD) [20] and Adam [21] as an optimization algorithm to update the network weights. The comparison shows that the Adam optimizer outperforms the prediction of wine quality than Gradient descent, so we use Adam as an optimization algorithm and the optimized network that can make the best prediction is obtained. The detailed architecture and the working of ANNs can be found elsewhere. In this work, we use ANN with one input layer, three hidden layers (each with 15 neurons) and one output layer.

For the training and test process, we choose a 60-20-20 train-validation-test split. Before passing to the network for the training purpose, the data were normalized by using the method described earlier in Equation (1). The model was trained on the training set and validated on the validation set to make sure there is no overfitting or underfitting during the training process. By tuning various hyperparameters such as learning rate, batch size, and number of epochs an optimized ANN model is obtained. Once, the model is optimized, it is tested on the test dataset. and its performance is evaluated by using MSE, R and MAPE. The performance comparison between various mathematical models and the ANN used in this work is presented in Table 3.

As presented in Table 3, GBR model shows the best performance (highest $\mathrm{R}$ as well as least MSE and MAPE) among the four models we used to predict the wine quality. The performance of ANN is very close to other models, but it is unable to surpass the accuracy obtained for GBR. It might happen because of the small number of datasets, we used to train the ANN, or the dataset is too simple, and the model is complex to learn enough the data. In addition, importance features from GBR that determines the wine quality is presented in Figure 6. When we plot the feature importance of all features for our GBR model we see that the most important feature to control the wine quality is turn out to be an alcohol. Which perfectly make sense because it is not only about the feelings after drinking in fact it effects the teste, texture and structure of the wine itself. The second

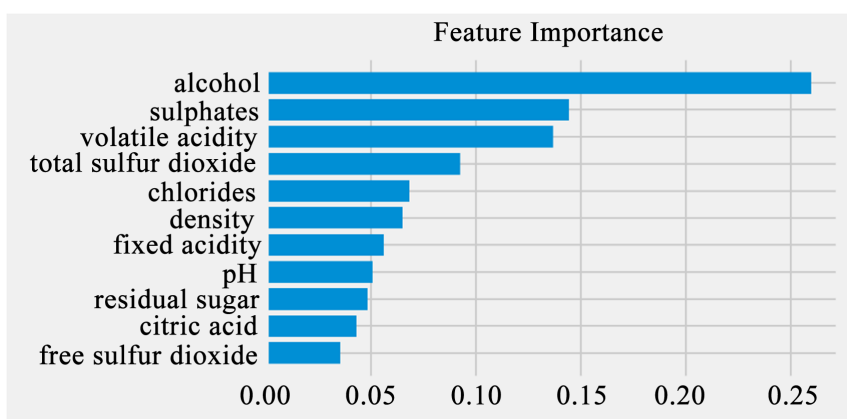

Figure 6. Feature importance for the wine quality for our best model GBR. 
most important feature is the sulphates, which is by definition somewhat correlated with the first feature. From plot what we also observed is the least important feature is the free sulfur dioxide. Which is a measure of the amount of $\mathrm{SO}_{2}$ (Sulfur Dioxide) which is used throughout all stages of the winemaking process to prevent oxidation and microbial growth [22].

\section{Conclusion}

This work demonstrated that various statistical analysis can be used to analyze the parameters in the existing dataset to determine the wine quality. Based on various analysis, the wine quality can be predicted prior to its production. Our work shows that among various ML models, Gradient Boosting performs best to predict the wine quality. The prediction of ANN lies behind other mathematical models; this is reasonable in such a small and heavily skewed dataset with the possibility of many outliers. Even though Gradient Boosting showed better performance, if we are able to increase the training datasets, then we might be able to get the benefits of prediction performance of ANN. This work shows an alternative approach that could be used to get the wine quality and, hence it can be a good starting point to screen the variables on which the wine quality depends.

\section{Conflicts of Interest}

The authors declare no conflicts of interest regarding the publication of this paper.

\section{References}

[1] Li, H., Zhang Z. and Liu, Z.J. (2017) Application of Artificial Neural Networks for Catalysis: A Review. Catalysts, 7, 306. https://doi.org/10.3390/catal7100306

[2] Shanmuganathan, S. (2016) Artificial Neural Network Modelling: An Introduction. In: Shanmuganathan, S. and Samarasinghe, S. (Eds.), Artificial Neural Network Modelling, Springer, Cham, 1-14. https://doi.org/10.1007/978-3-319-28495-8 1

[3] Jr, R.A., de Sousa, H.C., Malmegrim, R.R., dos Santos Jr., D.S., Carvalho, A.C.P.L.F., Fonseca, F.J., Oliveira Jr., O.N. and Mattoso, L.H.C. (2004) Wine Classification by Taste Sensors Made from Ultra-Thin Films and Using Neural Networks. Sensors and Actuators B: Chemical, 98, 77-82. https://doi.org/10.1016/j.snb.2003.09.025

[4] Cortez, P., Cerdeira, A., Almeida, F., Matos, T. and Reis, J. (2009) Modeling Wine Preferences by Data Mining from Physicochemical Properties. Decision Support Systems, Elsevier, 47, 547-553. https://doi.org/10.1016/j.dss.2009.05.016

[5] Larkin, T. and McManus, D. (2020) An Analytical Toast to Wine: Using Stacked Generalization to Predict Wine Preference. Statistical Analysis and Data Mining. The ASA Data Science Journal, 13, 451-464. https://doi.org/10.1002/sam.11474

[6] Lin, E.B., Abayomi, O., Dahal, K., Davis, P. and Mdziniso, N.C. (2016) Artifact Removal for Physiological Signals via Wavelets. Eighth International Conference on Digital Image Processing, 10033, Article No. 1003355. https://doi.org/10.1117/12.2244906

[7] Dahal, K.R. and Mohamed, A. (2020) Exact Distribution of Difference of Two Sample Proportions and Its Inferences. Open Journal of Statistics, 10, 363-374. https://doi.org/10.4236/ojs.2020.103024 
[8] Dahal, K.R., Dahal, J.N., Goward, K.R. and Abayami, O. (2020) Analysis of the Resolution of Crime Using Predictive Modeling. Open Journal of Statistics, 10, 600610, https://doi.org/10.4236/ojs.2020.103036

[9] Crookston, N.L. and Finley, A.O. (2008) yaImpute: An R Package for kNN Imputation. Journal of Statistical Software, 23, 1-16. https://doi.org/10.18637/jss.v023.i10

[10] Dahal, K.R. and Gautam, Y. (2020) Argumentative Comparative Analysis of Machine Learning on Coronary Artery Disease. Open Journal of Statistics, 10, 694-705. https://doi.org/10.4236/ojs.2020.104043

[11] Caruana, R. and Niculescu-Mizil, A. (2006) An Empirical Comparison of Supervised Learning Algorithms. Proceedings of the 23rd International Conference on Machine Learning, June 2006, 161-168.

https://doi.org/10.1145/1143844.1143865

[12] James, G., Witten, D., Hastie, T. and Tibshirani, R. (2013) An Introduction to Statistical Learning: With Applications in R. Springer, Berlin, Germany.

[13] Joshi, R.P., Eickholt, J., Li, L., Fornari, M., Barone, V. and Peralta, J.E. (2019) Machine Learning the Voltage of Electrode Materials in Metal-Ion Batteries. Journal of Applied Materials, 11, 18494-18503. https://doi.org/10.1021/acsami.9b04933

[14] Friedman, J.H. (2001) Greedy Function Approximation: A Gradient Boosting Machine. Annals of Statistics, 29, 1189-1232. https://doi.org/10.1214/aos/1013203451

[15] Chen, C.M. Liang, C.C. and Chu, C.P. (2020) Long-Term Travel Time Prediction Using Gradient Boosting. Journal of Intelligent Transportation Systems, 24, 109 124. https://doi.org/10.1080/15472450.2018.1542304

[16] Turian, J.P., Bergstra, J. and Bengio, Y. (2009) Quadratic Features and Deep Architectures for Chunking. Human Language Technologies Conference of the North American Chapter of the Association of Computational Linguistics, Boulder, Colorado, 31 May-5 June 2009, 245-248.

[17] Nwankpa, C., Ijomah, W., Gachagan, A. and Marshall, S. (2018) Activation Functions: Comparison of trends in Practice and Research for Deep Learning. arXiv: 1811.03378

[18] Nair, V. and Hinton, G.E. (2010) Rectified Linear Units Improve Restricted Boltzmann Machines. Proceedings of the 27 th International Conference on International Conference on Machine Learning, June 2010, 807-814.

[19] Glorot, X., Bordes, A. and Bengio, Y. (2011) Deep Sparse Rectifier Neural Networks. Proceedings of the Fourteenth International Conference on Artificial Intelligence and Statistics, 15, 315-323.

[20] Amari, S. (1993) Backpropagation and Stochastic Gradient Descent Method. Neurocomputing, 5, 185-196. https://doi.org/10.1016/0925-2312(93)90006-O

[21] Kingma, D.P. and Ba, J.L. (2014) Adam: A Method for Stochastic Optimization. arXiv: 1412.6980

[22] Monro, T.M., Moore, R.L., Nguyen, M.C., Ebendorff-Heidepriem, H., Skouroumounis, G.K., Elsey, G.M. and Taylor, D.K. (2012) Sensing Free Sulphur Dioxide in Wine. Sensors, 12, 10759-10773. https://doi.org/10.3390/s120810759 